«À quel point dépend je de moi-même? » Anxiété et corps selon Maine de Biran

\title{
Luís António Umbelino
}

University of Coimbra

lumbelino@fl.uc.pt

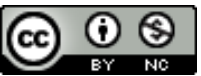

\section{Résumé}

Dans cet article, nous souhaitons méditer sur la modernité de Maine de Biran en ce qui concerne la philosophie de l'anxiété. Biran note d'abord un lien entre l'anxiété et la motilité ; deuxièmement, Biran nous invite à penser que l'intersubjectivité n'est pas étrangère à la sensation d'anxiété. Troisièmement, Biran se sent « surpris à plusieurs reprises », comme si sa propre anxiété, bien qu'intérieure, semblait provenir de l'extérieur. Ce sont les axes du travail que nous présentons ici.

Mots-clé: Anxiété, corps, affectivité, espace, Maine de Biran.

\begin{abstract}
“'To What Extent Can I Depend on Myself?'Anxiety and Body in Maine de Biran”

In this paper I aim to reflect on Maine de Biran's modernity with regard to the subject of a philosophy of anxiety. Biran first notes a connection between anxiety and motility; secondly, Biran invites us to think of intersubjectivity as something that is not foreign to the sensation of anxiety; third, Biran feels "surprised several times", as if his own anxiety, while interior, were something that seems to originate from outside. These will by the main axes of this paper.
\end{abstract}

Keywords: Anxiety, body, affectivity, space, Maine de Biran. 


\section{De l'anxiété selon Maine de de Biran}

Le 30 juillet 1816, le Questeur de la chambre des députés avait passé une mauvaise journée. Comme une harpe éolienne, on dirait que ses nerfs correspondaient au temps nébuleux et froid, au vent et à la pluie des Pyrénées, où il se trouvait dans un petit spa à la mode. Après le bain et le repos du matin, le Questeur, nommé Maine de Biran, politicien fameux, philosophe connu de quelques esprits innovateurs et attentifs de son temps, essaie de s'occuper de sa correspondance. Il le fait avec de considérables efforts, se battant contre des obstacles internes qui le dérangent. Il termine sa tâche laborieusement et tente d'écrire quelques notes dans son Journal intime. Son malaise nerveux persiste : les lignes sur le papier commencent à disparaître, ses yeux tremblent, son estomac vibre comme si des fourmis folles l'envahissaient. La concentration l'abandonne complètement. Il appelle un laquais et lui demande de préparer son cheval. Peut-être une promenade à cheval permettrait-elle de retrouver un peu l'équilibre de sa «pauvre machine » (Maine de Biran, 1954-57 : I, 186). C'est le moment où Madame la Duchesse de Rohan, accompagnée de son collègue Castel-Bajac, frappe à la porte de notre philosophe au sujet d'une quête pour les pauvres de Saint-Sauveur.

Voici alors ce qui s'est passé, tel que Maine de Biran l'a rapporté dans son Journal à la fin de la journée :

Je savais que cette quête devait avoir lieu, et j'avais prédéterminé six francs, croyant que ce serait là le taux commun des baigneurs aisés. J'ai donc mis mon écu dans la bourse de la quêteuse, avec un peu d'embarras et doutant si je faisais ce qui convenait : j'ai eu la certitude du contraire, lorsque la duchesse m'a cité des personnes, qui avaient donné un ou deux louis. Cette parole m'a bouleversé, et j'ai éprouvé à l'instant une agitation d'esprit et un regret aussi vif que si j'avais commis la plus mauvaise et la plus déshonorante de toutes les actions. Je n'ai pas eu la force [...] de dire un seul mot [...]. Dès ce moment, il m'a été impossible de songer à autre chose [...]. Je cherchais vainement à me distraire, et je portais sans cesse ce poids sur mon cœur [...] cette idée, fatigante par sa fixité [...]. J'ai été me promener à pied devant sa maison [de la duchesse] [...] mais j'ai craint de faire encore une gaucherie. [...] Je suis rentré pour diner avec la même anxiété. Mon idée fixe allait au point de me faire parler, gesticuler tout seul. Je me suis surpris plusieurs fois 
dans cet état voisin de la démence [...]. (Maine de Biran, 1954-57 : I, 186187)

Ce qui m'intéresse dans cette description ce n'est pas le cas personnel de Biran, mais le fait que le philosophe, dans son Journal, se propose de méditer philosophiquement sur cet état que s'abat régulièrement sur lui. Il l'interroge, précisément, en tant que philosophe. Et, en ce qui concerne l'anxiété, il se pose cette question décisive et qui dit presque tout : «À quel point dépends-je de moi-même ? » (Maine de Biran, 1954-57 : III, 166). Encore plus, au moment où il semble toucher le cas particulier d'anxiété qu' on nomme « agoraphobie », nous retrouvons, encore dans son Journal, la question la plus radicale :

Qu'est-ce qui m'oblige à tant me remuer, à aller d'un objet à l'autre ? Pourquoi consens-je à perdre dans ce misérable jeu toute la gravité qui me convient, tout sérieux de la vie !... Tout mon mal vient de ne pas savoir me tenir tranquille dans ma chambre à Paris, comme à la campagne, et ne savoir rester maître de mon propre mouvement. (Maine de Biran, 1954-57 : I, 42)

Notons, avant de poursuivre, l'actualité de Biran :

a) Biran remarque d'abord une connexion entre l'anxiété et la motilité. Ça se confirme d'une façon paradigmatique dans le cas de l'agoraphobe : l'anxiété est quelque chose qui, soit inhibe des mouvements du corps par une sorte de fixité aveugle qui nous «absorbe » et paralyse, soit mobilise le corps (toujours aveugle), soudainement pris de la nécessité de changer de lieu sans volonté et sans but, de fuir, ou de « gesticuler tout seul ». L'anxieux se met soit en mouvement pour fuir, soit il s'arrête à une décision qu'il ne prend pas vraiment.

b) Deuxièmement, Biran nous invite à penser que la dimension intersubjective de notre façon d'être au monde n'est pas étrangère à la sensation d'anxiété en des contextes sociaux. Encore une fois, il a raison: sous l'influence du regard de l'autre, sous l'influence du volume de son corps ou de ses mots, l'anxieux agoraphobe peut tomber «craintif et troublé dans la moindre chose, voyant partout [au dehors] des dangers » et des menaces de mort (Gallagher et Trigg, $2016: 6$ ). 
c) Troisièmement, Biran se sent « surpris plusieurs fois », comme si sa propre anxiété, tout en étant à l'intérieur de l'intérieur, venait $d u$ dehors. Tout se passe pour Biran comme si l'anxiété - la sienne même - venait d'une sorte de fatum $^{1}$ du corps opérant en nous sans nous. Voilà un point décisif et plein d'actualité : le corps de l'anxieux est bien ce que Biran nommait le corps affectif, un corps si archaïque que vivant, si aveugle que promoteur de la variabilité confuse de notre façon de sentir l'existence, si sauvage que prometteur du ton et des couleurs atmosphériques qui baignent l'accès au monde et aux autres, si inconnu dans ses racines que constitutif de nos alternances tempéramentales. Un tel corps - qui demeure le sien - apparaît à l'anxieux comme une chose, comme - dirait Biran - un corps qui résiste à consister dans l'effort aperceptif, qui résiste à devenir corps propre. Dans l'agoraphobie, en fait, le corps apparaît comme une chose avec un pouvoir immense de réfraction sur la conscience, et non comme corps propre ou corpssujet, c'est-à-dire, comme centre du soi agissant.

d) Finalement, la surprise de notre philosophe est de se trouver soudainement dans un état «voisin de la démence ». Là encore, il a bien vu l'essentiel : les dispositions internes de l'affectibilité - qui donnent le ton du sentiment de l'existence - sont les mêmes qui peuvent arriver à «absorber » (Maine de Biran, 1954-57 : II, 123-124) le moi, à dissoudre la possession de soi, à usurper la ligne de la conscience ; une frontière poreuse se signale, donc, dans l'agoraphobie, une frontière devant laquelle on pressent la possibilité et la menace d'annulation totale de la possession de soi. D'un point de vue philosophique, voici le plus important : l'anxiété (notamment agoraphobique) n'est pas simplement le dérangement des impressions ou des symptômes physiques, mais la menace que ces symptômes aveuglement corporels, intersubjectifs et spatialisés, posent à l'intégrité du régime de possession de soi.

\footnotetext{
${ }^{1}$ Maine de Biran, 1984b : $3:$ in mente est providencia, in corpore est fatum; Maine de Biran $(2001: 214)$ : «N'est-il pas en effet comme le destin, cet agent invisible de la vie qui opère en nous sans nous et dont nous subirions toujours les lois, alors même que ce qui est le fatum dans le physique deviendrait prévoyance dans le moral ?».
} 


\section{Un corps agoraphobique?}

Si on prend au sérieux ce qui vient d'être dit, il faut donc conclure que Biran anticipe l'occasion d'inventorier les limites soit dans l'approche strictement clinique de l'anxiété agoraphobique, soit dans l'approche strictement causale et cognitive, soit dans l'approche strictement neurophysiologique.

Dans un texte récent, Shaun Gallagher et Dylan Trigg nous ont montré le plus décisif à ce propos (Gallagher et Trigg, $2016: 6$ ) : selon l'approche clinique, nous reconnaissons les symptômes : palpitations, rythme cardiaque sans contrôle, crise de panique (attaque de panique), peur anticipée d'une attaque de panique, tremblement à l'estomac, faiblesse aux jambes, nausée, crainte de devenir fou, sensation de tragédie imminente, étrangeté du corps, sensation d'aliénation d'une partie de soi-même, comportements d'évitement des situations angoissantes, incapacité de se mouvoir ou comportements de fuite, etc. ; selon l'approche causale, on peut reconnaître : (a) des fautes de la pensée qui nous font, d'une façon dérèglée, reconnaître des menaces des environnements au moment de la réponse phobique; (b) des associations d'idées sans contrôle que suivent une telle reconnaissance; (c) des interprétations équivoquées de tout ce que nous percevons comme des symptômes bizarres et sauvages de notre corps ; (d) des comportements qui sont mis en place en anticipant le retour de l'anxiété et qui ne sont que des stratégies pour éviter les sensations indésirables ; (e) des fonctions que l'on peut trouver subjacentes à l'expérience phobique et qui peuvent nous aider à comprendre le rôle de la peur elle-même dans la vie humaine (Shawn, 2007 : 227 ; Gallagher et Trigg, $2016: 7)$. Ce que l'on nomme aujourd'hui oriented behavioral therapy (CBT) témoigne de l'importance d'un tel travail d'identification. Finalement, c'est aussi vrai que les développements de l'approche neuroscientifique de l'agoraphobie nous ont appris des aspects inédits, notamment, ceux qui concernent les corrélats neuronaux visant à cartographier les dynamiques du cerveau et à prédire des nouveaux traitements par la voie d'une espèce de reprogrammation des connexions neuronaux. Tout ce que vient d'être dit est d'importance. Mais il faut encore ajouter que ces approches restent insuffisantes pour une exposition complète du phénomène de l'anxiété - notamment de l'anxiété agoraphobique (Gallagher et Trigg, 2016 : 7). Il manque l'étude, précisément, de ce que Biran nous a suggéré et que la 
phénoménologie contemporaine nous aide à penser : les connexions coconstitutives de la spatialité vécue, de la corporalité anonyme (affectif, spatialisée et intersubjective) et du développement toujours menacé du régime de possession de soi (ce que l'on appelle aujourd'hui, par exemple, sens of agency et sense of ownership : Gallagher et Trigg, $2016: 2-10)$ sous le pouvoir d'effraction de l'affectivité.

\section{Les paysages affectives et le fatum du corps}

Considérons d'abord l'évidence selon laquelle l'anxieux « est lié intimement à la sensation d'un manque de contrôle sur nos sentiments et nos actions » (Capps et Ochs, 1997, cit. in Gallagher et Trigg, 2016 : 8), au sentiment dérangeant de la possibilité de perdre ou dépossession de soi. Biran l'avait bien compris avec sa distinction entre corps propre et corps affectif: selon le philosophe de Bergerac, le corps propre est le corps de la résistance docile à l'intérieur de l'effort primitif, le corps qui offre le plan de consistance persévérante à la volonté, celui-ci étant donc un élément constitutif du fait de conscience luimême. Au lieu du cogito cartésien - qui en tant que substance qui pense, doit penser toujours -, Biran parle d'un volo, d'un « je peux » corporalisé, d'un « je suis » qui pense seulement avec son corps. Or, si je pense toujours avec le corps, il faut ajouter que je ne pense pas toujours. Le sujet biranien est bien celui qui, en découvrant la constitution corporelle de la pensée, fait en même temps l'expérience incarnée de la fragilité d'un tel acte. Pour Biran, ça s'explique par le fait que notre corps propre n'est pas tout ce que je suis en tant que corps. Ma corporéité fait figure d'une interface: soit elle se dispose docilement à devenir corps propre (l'élément constitutive du fait primitif de conscience, du moi aperceptive, de la possibilité de se posséder soi-même), soit la corporéité montre sa face déragent, fluide, sauvage, dépossédant - sa face affective qui est l'emblème de notre impuissance vis-à-vis les alternances du fatum.

Biran nous parle ici du « corps affectif », le corps du flux de l'affectivité qui opère en nous sans nous ${ }^{2}$ (c'est-à-dire: qui reste hors le rapport d'effort

\footnotetext{
${ }^{2}$ Voir Maine de Biran, 1984b : 24. Maine de Biran, $2001: 214:$ « N'est-il pas en effet comme le destin, cet agent invisible de la vie qui opère en nous sans nous et dont nous
} 
aperceptive, mais le hante régulièrement comme si venant d'un dedans de nous qui est un dehors du moi) et nous donne le ton du sentiment d'existence comme un voile ; un voile

qui nous montre la nature extérieure tantôt sous un aspect riant et gracieux, tantôt comme couverte d'un voile funèbre, qui nous fait voir dans les mêmes choses, les mêmes êtres, tantôt des objets d'espérance et d'amour, tantôt des sujets de méfiance et de craint. (Maine de Biran, 1984a : 29)

Sous la densité corporelle résistante, le flux des affections me révèle toujours que même dans la plus haute maîtrise sur mon corps je ne me donne pas mon corps. Le corps affectif, avec ses lois aveugles, nous détermine «sans se savoir » (Maine de Biran, 1984b : 24). Et Biran d'ajouter une remarque si surprenante que décisive: concernant la racine d'un tel flux secret des affections - « la source de presque tout le charme ou le dégoût attaché aux divers instants de notre vie » (Maine de Biran, 1988 : 92) -, nous la portons en nous, même si leur cause demeure pour nous inconnue ; « Eh ! qu'importe en effet que cette puissance inconnue soit en nous ou hors nous ?» (Maine de Biran, 1988 : 92 ; Maine de Biran, 2001 : 214).

Il y dans ces remarques de Biran une double thèse qui reste d'actualité (en accord avec l'approche phénoménologique contemporain du thème de l'anxiété). D'abord la thèse selon laquelle il a une dimension de notre corporéité - sauvage, affective, anonyme - qui correspond à une façon d'apparaitre de « notre » corps à la manière d'une chose et comme chosifié. Plus précisément, dans l'affectivité, selon Biran, «mon» corps m'apparaît comme étranger, comme différent, ce qui ne veut pas dire, bien sûr, qu'il faut parler ici de n'importe quelle forme de dualisme substantialiste (Gallagher et Trigg, 2016 : 8 ; Trigg, 2012). Bien au contraire : ce qui se passe dans ce cas c'est que le corps esquisse affectivement une espèce de réfraction para rapport au moi aperceptif et, en même temps, d'effraction dérangeante sur la vie de conscience

subirions toujours les lois, alors même que ce qui est le fatum dans le physique deviendrait prévoyance dans le moral ?». 
qui s'annonce, à la limite, comme une espèce de possibilité vécue d'absorption $\mathrm{du}$ «moi » parque ce que, « en mois », n'est pas $d u m o i^{3}$

D'un point de vue phénoménologique, cette idée d'un apparaitre du corps comme corps étrangère est décisive. Notre expérience d'être corps est telle que nous avons besoin, à chaque moment, de «percevoir » expressément le corps pour avoir un monde et agir au monde. C'est que le corps, en tant que notre seul « véhicule d'être au monde » (Merleau-Ponty, 1945 : 294-295), reste ce qui nous offre - comme s'il était une force d'imbriquement intentionnel nonintentionné - l'occasion d'être au monde sans que aucun effort réflexif de la pensée abstraite ne soit nécessaire. Un tel corps n'est pas compris comme un assemblage d'organes juxtaposés dans l'espace, mais comme une totalité d'appartenance ou de connivence mondaine. C'est dans ce sens que le corps demeure dans l'espace, comme disait Merleau-Ponty, «comme le cœur dans l'organisme : il maintient continuellement en vie le spectacle visible, il l'anime et le nourrit intérieurement, il forme avec lui un système » (Merleau-Ponty, 1945 : 235); et il le fait dans le silence et l'anonymat d'une motricité qui signifie par appartenance. Ainsi, chaque oscillation et mouvement du corps basculent toujours au rythme de l'espace qui le fait être de quelque part. L'espace est l'autre côté du système du corps que je suis sans le « percevoir ». Or, ce qui se passe c'est qu'il y a des circonstances où une telle façon d'être du corps, façon tacite d'être vocation irréfléchie de monde, disparait - ou disappear, comme disait Drew Leder (Leder, 1990). C'est le cas, par exemple, d'une douleur intense : le corps silencieux disparaît et à sa place apparaît un corps identifiable dans ses parties concrètes comme une chose étrange qui m'empêche de fuir la douleur (qui m'empêche de fuir moi-même en train de me faire mal sans contrôle). Le corps souffrant est un corps étranger et étrange, un corps autre, chosifié, et pourtant affectivement connecté à «moi ». Pour la plupart des cas, cette circonstance est momentanée et n'a pas d'implications sur le tout et le fonds de nos capacités perceptives et motrices. Néanmoins, quand on parle d'anxiété (notamment en partant du cas de l'agoraphobie) « c'est une autre histoire » (Gallagher et Trigg, $2016: 8$ ) et quelque chose de surprenant

\footnotetext{
${ }^{3}$ Le « psychologique » et le «physiologique » cherchant à se mixer dans des nouvelles combinaisons imprévues.
} 
nous est révélé des connexions entre l'expérience de la chosification ou de l'aliénation du corps et le « je peux » spatialisant-spatialisé :

différemment de l'expérience normale de la spatialité et du sens de l'agir, dans laquelle le corps offre au monde un je peux, un centre rassuré d'orientation activement engagé dans les sollicitations de ce qui l'entoure, l'expérience incarnée de l'espace et de l'action dans l'espace effectuée par l'agoraphobe est caractérisée par l'hésitation, le malaise, et par la perte de confiance dans ses propres capacités pour répondre à une situation non familière. (Gallagher et Trigg, $2016: 8)^{4}$

Cela nous fait penser à la deuxième thèse décisive de Biran, dont découle de la suggestion suivante : «peu importe » que la source sécrète des affections soit en nous ou hors nous. C'est une affirmation remarquable ; l'intimité des affections reste, pour Biran, question - comme l'a bien montré Marc Richir d'une l'Innenleiblichkeit (Richir, 2000); bien qu'ancrée dans le corps de « l'organisation », elle n'est pas, en rigueur, dans « le corps » du physiologiste ou de l'anatomiste, où l'affectivité n'arriverait jamais à être expliquée. Et pourtant, on sait que les affections bougent et me font bouger à son propre rythme, me font pâtir d'une façon tempéramentale ou atmosphérique le monde et les autres; que son origine soit en moi ou hors moi peut import parce que sont des telles rythmes affectives, précisément, qui nous font voir le monde, les lieux et les autres tantôt sous un aspect riant et gracieux, tantôt comme couverte d'un voile funèbre, tantôt comme des objets d'espérance et d'amour, tantôt comme des sujets de méfiance et de crainte.

À la limite, donc, il fallait peut-être ajouter qu'au niveau de l'affectivité on est touché par une spatialité atmosphérique et intersubjective qui apparait avant la distinction classique du sujet et de l'objet. En fait, l'affectivité n'est pas, à proprement parler, « dans le sujet » (qui la pâte comme si elle venait du dehors, du fatum du corps) ; l'affectivité n'est non plus « du côté objectif de l'analyse physiologique (dans le sens ou l'affectivité demeure pouvoir d'effraction sur le plan de la «vie de relation ou conscience »). Et, pourtant, l'affectivité marque notre premier rapport au monde et aux autres, mixant les propriétés des lieux et

\footnotetext{
${ }^{4}$ Nous traduisons.
} 
l'orbite sympathique des outres avec les variations d'humeur que nous portons en nous sans nous.

L'anxiété agoraphobique que Biran interroge en philosophe c'est, dans ce sens, bien une «autre histoire » du corps : l'anxieux fait l'expérience du chosifiable du corps en tant qu'oscillation aveugle qui peut devenir une inhibition ; une inhibition qui, d'une certaine façon, semble se compléter dans un espace intersubjectif devenu lui-aussi, dans quelques de ses régions, chosifiable, étranger, aliénant, étrange - même si encore affectivement lié à «mon » corps. En même temps, le corps affectif devient aliéné par rapport au corps approprié qui reste le véhicule silencieux (originairement intentionnel, dynamiquement engagé) de notre être au monde et, avec lui, l'espace atmosphérique apparait lui-aussi à l'anxieux comme aliéné et aliénant (et capable d'absorber le moi, comme disait Biran) de la familiarité du monde.

Biran avait donc raison au moment où il suggère que l'anxiété garde une attention monopolisatrice à des sensations et des mouvements qui ne semblent venir d'aucune part, c'est-à-dire, qui semblent venir du fatum du corps, d'un corps autonome (plus que du «moi persévérant» de l'effort). À la limite du régime de possession de soi, le trouble d'anxiété reste donc le témoigne compatissant et souffrant des rythmes du corps, de la vie, de l'espace, du monde, des autres, s'exerçant en nous sans nous.

\section{Bibliography}

CAPPS, L. et E. OCHS (1997). Constructing Panic. The Discourse of Agoraphobia. Boston, MA : Harvard University Press.

Gallagher, Sh. et D. TRIGG. (2016). «Agency and Anxiety : Delusions of Control and Loss of Control in Schizophrenia and Agoraphobia ». In: Frontiers in Human Neurosciences, 10: 459, pp. 1-12. <DOI: 10.3389/fnhum.2016.00459>

LEDER, D. (1990). The Absent Body. Chicago : University of Chicago Press.

MAINE DE BIRAN (2001). Essai sur les fondements de la psychologie II. In : Euvres de Maine de Biran, t. VII-2. Paris : Vrin.

- (1988). Mémoire sur la décomposition. In : Euvres de Maine de Biran, t. III. Paris : Vrin. 
- (1984a). Discours à la société médicale de Bergerac. In : Cuvres de Maine de Biran, t. V. Paris : Vrin.

- (1984b). Rapports du physique et du moral de l'homme. In: Euvres de Maine de Biran, t. VI. Paris : Vrin.

- (1954-57). Journal I-III. Paris : Éditions de la Baconnière.

MERleAu-PONTY, M. (1945). Phénoménologie de la perception. Paris : Gallimard.

RICHIR, M. (2000). "Stimmung, Verstimmung et Leiblichkeit dans la schizophrénie ». In : AAVV. Conferências de Filosofia II. Porto : Campo das Letras, 57-69.

SHAwn, A. (2007). Wish I Could be There. Notes from a Phobic Life. New York : Viking.

TRIGG, D. (2012). The Memory of Place. Phenomenology of the Uncanny. Athens, Ohio : Ohio University Press.

Luís ANTÓNIO UMBELINO holds a $\mathrm{PhD}$ in philosophy. He is Professor at the Faculty of Arts, University of Coimbra. He is a member of the research unit "CECH - Centro de Estudos Clássicos e Humanísticos" (FCT-Portugal) and of the research project "Fenomenología del cuerpo y análisis del dolor II" (FFI 2017-82272-P - Spain). He is director of the journal Revista Filosófica de Coimbra. He is the author of several international publications focused, in particular, on Maine de Biran, French phenomenology (Merleau-Ponty), and philosophical hermeneutics (Ricoeur).

LUIS ANTONIO UMBELINO est docteur en philosophie et professeur auxiliaire à la Faculté des Lettres de l'Université de Coimbra. Il est chercheur dans l'unité de recherche «CECH - Centre d'Études Classiques et Humanistiques » (FCTPortugal) et membre du projet de recherche «Fenomenología del cuerpo y análisis del dolor II » - FFI 2017-82272-P - Espagne. Directeur de la Revista Filosófica de Coimbra, il a publié de nombreux articles, au Portugal et à l'étranger, sur la tradition réflexive française (Maine de Biran), sur la phénoménologie française (Merleau-Ponty) et sur l'herméneutique philosophique (Ricœur). 